\title{
O experimento WS de 1950 e as suas implicações para a segunda revolução da mecânica quântica
}

The WS experiment of 1950 and its implications for the second revolution of quantum mechanics

\author{
Angevaldo Menezes Maia Filho*1잉, Indianara Silva ${ }^{1,2}$ \\ ${ }^{1}$ Universidade Federal da Bahia, Instituto de Física, Programa de Pós-Graduação em Ensino, Filosofia e História das Ciências, \\ Salvador, BA, Brasil \\ ${ }^{2}$ Universidade Estadual de Feira de Santana, Departamento de Física, Feira de Santana, BA, Brasil
}

Recebido em 16 de Junho, 2018. Aceito em 03 de Novembro, 2018.

\begin{abstract}
Neste trabalho, discute-se o papel do experimento de 1950 realizado pela física sino-estadunidense Chien Shiung $\mathrm{Wu}$, em colaboração com seu assistente de pesquisa, Irving Shaknov, nas discussões sobre os fundamentos da teoria quântica. O trabalho citado, que ficou conhecido como experimento WS, é comumente apontado como sendo o primeiro experimento realizado capaz de representar o fenômeno que hoje é conhecido como entrelaçamento quântico, tornando-se a chave para o desenvolvimento de novas tecnologias, tais como, a criptografia, a teleportação e a computação quântica. Esse experimento garante a Wu um papel fundamental no que diz respeito à corroboração da Segunda Revolução Quântica, tornando-se ainda um episódio importante para garantir a visibilidade das mulheres na história da física e da teoria quântica.
\end{abstract}

Palavras-chave: Chien Shiung Wu, Mulheres na Ciência, História da Mecânica Quântica, História da Física, História das Mulheres.

In this paper, we discuss the role of the 1950 experiment conducted by Sino-American physics Chien Shiung Wu in collaboration with his research assistant, Irving Shaknov, in the discussions about the foundations of quantum theory. The quoted work, known as the WS experiment, is commonly referred to as the first realized experiment capable of representing the phenomenon that is now known as quantum entanglement, becoming the key to the development of new technologies such as cryptography, teleportation and quantum computing. This experiment guarantees $\mathrm{Wu}$ a fundamental role in corroborating the Second Quantum Revolution, and is still an important episode to ensure the visibility of women in the history of physics and quantum theory.

Keywords: Chien Shiung Wu, Women in Science, History of Quantum Mechanics, History of Physics, History of Women.

\section{Introdução}

Ao pensarmos em teorias físicas, a teoria quântica é uma das que possui maior prestígio na comunidade científica. Além da sua contribuição para o avanço e desenvolvimento tecnológico da humanidade, trata-se ainda de uma das teorias mais bem-sucedidas da história da física. Tendo o próprio desenvolvimento da mecânica quântica (MQ) na década de 20 enquanto uma revolução no estudo do tema, a segunda revolução quântica, termo cunhado pelo físico experimental Alain Aspect, trouxe ainda mais contribuições para o desenvolvimento da área a partir do aprimoramento de técnicas experimentais na década de 60 , inclusive para o desenvolvimento do campo de pesquisa da informação quântica [1]. Ainda nos dias atuais, a MQ é um dos campos de pesquisa mais promissores no que diz respeito a novas tecnologias e discussões teóricas na compreensão dos fenômenos físicos.

*Endereço de correspondência: angevaldomaia@gmail.com
A MQ teve o seu desenvolvimento na primeira metade do século XX com grandes físicos como protagonistas, a saber, Max Planck, Niels Bohr, Werner Heisenberg, Louis de Broglie, Erwin Schrödinger e Albert Einstein. Estes são os nomes mais conhecidos e que, muitas vezes, carregam ainda o título de fundadores ou pais da física quântica - o que evidência o impacto positivo destes físicos na criação da mecânica quântica [2]. Contudo, há muitos outros personagens, alguns até desconhecidos, que também contribuíram para as discussões sobre os fundamentos da MQ. Como bem destaca o historiador da ciência Massimiliano Badino [3], mesmo cientistas pouco convencionais podem ter contribuições extremamente significativas para diversas áreas da ciência.

Esse reconhecimento pode ser percebido, portanto, através do produto da pesquisa de historiadores da ciência que buscam, para além do campo da história intelectual (sustentada predominantemente nas teorias e nas equações), elementos e personagens considerados periféricos ao mainstream, de um determinado tema ou linha 
de pesquisa, para compor a sua narrativa. Em relação à teoria quântica, Badino [3] usou o termo quantum underground para caracterizar personagens invisibilizados pela história convencional da física. Por sua vez, Olival Freire [1], também historiador da ciência, utilizou o termo quantum dissidents para se referir a pesquisadores que se debruçaram sobre temas pouco convencionais da mecânica quântica e, com isso, foram capazes de desenvolver um campo de pesquisa extremamente importante para a física atual, a exemplo da informação quântica.

Compactuamos com o fato de que esses personagens e temas, ainda que por algum motivo invisibilizados, a partir de uma perspectiva histórica, podem apresentar contribuições tão significativas quanto a dos personagens e temas do considerado mainstream da física. A história usual da MQ é um campo protagonizado por homens em um contexto predominantemente europeu, o que é percebido nos grandes livros que tratam da história da mecânica quântica, a exemplo do Quantum Generations do historiador dinamarquês Helge Kragh [4]; e do The Philosophy of Quantum Mechanics do físico e filósofo da ciência alemão Max Jammer [5]. Diante disso, percebemos a necessidade de olhar para outras fontes e contribuições de modo a trazer à tona novos sujeitos e narrativas que podem estar localizados no quantum underground. Isso implica em resgatarmos na história da física personagens invisibilizados ou invisibilizadas, ou que tiveram sua trajetória pouco explorada em detrimento dos ditos grandes cientistas.

O não aparecimento, ou o tímido aparecimento, por exemplo, da contribuição das mulheres na evolução do pensamento sobre o tema da mecânica quântica requer uma análise crítica da história tradicional da física. No intuito de ir além das narrativas convencionais, resgatamos a contribuição do trabalho da física experimental sino estadunidense Chien Shiung Wu para os fundamentos da teoria quântica. O seu experimento de 1950 desencadeou o que ficaria posteriormente conhecido como a segunda revolução quântica, tornando-se o pioneiro na compreensão do fenômeno do entrelaçamento quântico que contribuiu para avanços nas áreas de teleportação quântica, criptografia quântica e computação quântica. Além disso, Wu também foi protagonista na realização de experimentos que comprovaram teorias físicas importantes, a exemplo da violação da paridade e da teoria de Fermi, bem como contribuiu com o estudo do decaimento beta.

Neste artigo, discutiremos de que forma o resultado experimental de Wu e Irving Shaknov (WS) contribuiu para as discussões dos fundamentos da mecânica quântica, e destacaremos a sua relevância para a segunda revolução quântica. Mais precisamente, o nosso foco é o desenvolvimento e a repercussão do experimento WS até o ano de 1957 quando os físicos David Bohm e Yakir Aharonov utilizaram-no como uma primeira possibilidade experimental para verificação do paradoxo EPR de 1935 [6,7]. A narrativa foi construída cronologicamente, iniciando com o trabalho EPR, seguido do experimento WS da década de 50, passando pelas discussões de Bohm sobre os fundamentos da teoria quântica e pelo teorema de Bell, e chegando finalmente nos experimentos da terceira geração que corroboraram a MQ.

Por fim, acreditamos contribuir para uma história das mulheres em áreas científicas, como a teoria quântica, predominantemente masculina, cuja historiografia tradicional desvaloriza ou pouco enfatiza o papel direto ou indireto de personagens do quantum underground como a própria $\mathrm{Wu}$.

\section{Chien Shiung Wu: um breve histórico da sua trajetória}

Chien Shiung $\mathrm{Wu}$ foi uma física experimental sino-estadunidense que contribuiu para diversas áreas da física. Com base na sua biografia escrita pelo jornalista Tsai Chien-Chiang, a sua boa reputação, enquanto física experimental e primeira mulher na presidência da American Physical Society (APS), legou a Wu pseudônimos utilizados no sentido de reconhecer a importância dos seus trabalhos, como, por exemplo, "a autoridade" no decaimento beta, a primeira dama da pesquisa em física, a pioneira em física nuclear e, até mesmo, a Marie Curie chinesa [8]; [9]. O seu legado também foi explorado por Richard Hammond em Chien Shiung Wu: Pionnering in Nuclear Physics. Apresentaremos, então, o capital científico da $\mathrm{Wu}$ que a tornou conhecida na comunidade científica e na sociedade.

A formação inicial de Wu aconteceu na China, onde obteve a sua graduação em física no ano de 1934 pela National Central University. Depois de graduada, trabalhou por um ano como assistente de ensino na Zhejiang University. Após esse período, Wu conseguiu o seu primeiro emprego na Academia Sinica, trabalhando com atividades experimentais em física e química, sob a orientação da cientista Gu Jing-Wei que havia realizado a sua pós-graduação nos EUA na Universidade de Michigan, e que acabou encorajando-a a continuar a sua formação. Wu chegou em 1936 nos EUA e realizou seus estudos de pós-graduação na Universidade de Berkeley. Foi orientada pelo Ernest Lawrence e trabalhou em um dos laboratórios mais modernos dos EUA. Ainda em Berkeley, Wu conviveu e tornou-se amiga de grandes nomes da física, como Emilio Segrè, Robert Oppenheimer e Wolfgang Pauli [8,9].

Wu participou do projeto Manhattan, um dos maiores projetos científicos do século passado, o qual contou com a contribuição das maiores mentes científicas da época. Trabalhou na obtenção de urânio físsil a partir do processo de difusão gasosa na Universidade de Columbia. Ainda nesse período, a tese de doutorado de $\mathrm{Wu}$ foi importante para auxiliar na resolução do problema no reator B que estava associado ao projeto $[8,9]$.

Após a guerra, Wu liderou uma equipe que foi capaz de apresentar em 1956 experimentalmente a violação de 
uma das propriedades de simetria considerada a mais fundamental na física: a paridade no decaimento beta. A sua importância foi tão grande e significativa que rendeu o Prêmio Nobel de 1957 para a proposta teórica dos físicos chineses Tsung Dao Lee e Chen-Ning Frank Yang $[8,9]$. Wu, no entanto, não foi incluída na premiação - o que se tornou um dos episódios mais representativos em relação à injustiça na premiação do Nobel envolvendo as questões de gênero. Ademais, realizou experimentos que corroboraram em 1948 a teoria de Fermi para o decaimento beta após mais de uma década de tentativas de outros físicos experimentais. Também se debruçou na área da biofísica contribuindo com estudos acerca da hemoglobina e anemia falciforme.

Em 1978, o prêmio de inauguração da premiação da Fundação Wolf foi escolhido por unanimidade para ser entregue a Wu por sua contribuição à exploração da interação fraca. O prêmio Wolf é o mais prestigiado da física depois do Nobel e é sustentado pela Fundação Wolf localizada em Israel desde 1976.

O experimento que apresenta a violação da paridade na física e a questão do não recebimento do prêmio Nobel, mesmo recebendo sete indicações, vinculado às questões de gênero, certamente garante um dos temas mais significativos a se tratar na vida de $\mathrm{Wu}$. No entanto, neste artigo, pretendemos compreender a relevância do experimento de 1950 na discussão acerca dos fundamentos e da segunda revolução da mecânica quântica, o qual foi negligenciado pelas principais biografias de $\mathrm{Wu}$ e pela literatura que tratam da história da MQ. Em uma análise macro, verificamos que, ao realizar o experimento, a preocupação de Wu estava aparentemente voltada para a verificação de resultados teóricos e experimentais associados à área da eletrodinâmica quântica. Ou seja, Wu não estava inserida na comunidade de físicos interessados nos fundamentos da mecânica quântica, mas, mesmo assim, o seu resultado experimental teve um impacto relevante na área.

A busca por experimentos capazes de corroborar teorias era uma das características mais marcantes e uma prática corriqueira do trabalho da nossa personagem, evidenciando, assim, a importância dada a uma prática científica preocupada tanto com a teoria quanto com a experimentação. Mesmo sendo realizado com outro objetivo que não abordar os fundamentos da teoria quântica, o experimento de Wu e Shaknov acabou sendo apropriado por Bohm para discutir a questão da não localidade.

Apesar do aparente distanciamento de áreas da física, institucionalmente fragmentadas, o historiador Christian Joas [10] argumenta que a mecânica quântica derivou de diversas áreas da física como, atualmente identificada, a física do estado sólido, física nuclear e física de partículas. Físicos, como o próprio Werner Heisenberg, se preocupavam com temas que envolviam teoria atômica, estrutura da matéria, turbulência de fluidos, relatividade, radiação atmosférica, radioatividade, entre outros, o que nos permite afirmar que a mecânica quântica não surge como uma área de estudo independente, e que o seu próprio amadurecimento contou com pesquisadores e pesquisadoras de áreas institucionalmente distintas. A história da MQ está entrelaçada com subdisciplinas da física que não tinham ainda identidade institucional.

\section{O argumento EPR}

Para compreendermos o papel do experimento de Wu, é importante apresentarmos brevemente um pouco da controvérsia existente a respeito das interpretações da MQ. A rigor, consideraremos duas das diversas interpretações da MQ: a de Copenhague - derivada da complementariedade de Niels Bohr publicada em 1928 [11] e que também ficou conhecida como usual ou ortodoxa, e a de David Bohm que trouxe à tona, em seu trabalho de 1952, discussões sobre o que ficou conhecido como "variáveis ocultas" as quais completariam a MQ [6], apresentando-se, assim, enquanto uma abordagem alternativa à interpretação usual.

A interpretação usual da MQ recebia questionamentos em relação à sua completude levando em consideração tanto conceitos físicos, a exemplo do problema da medição, quanto filosóficos. A interpretação de Copenhague defendia a MQ enquanto não determinística, apoiando-se em um caráter probabilista, por nomes como Niels Bohr, Max Born, Werner Heisenberg, Erwin Schrödinger, Wolfgang Pauli, e Léon Rosenfeld. Havia uma forte questão filosófica envolvida no debate em torno dos fundamentos da teoria quântica, o qual levou a uma controvérsia na comunidade científica quanto à pertinência ou não desta teoria ao realismo da física [12]. O critério de realidade, adotado pelos físicos que questionavam a MQ usual, consistia em considerar que se for possível prever o valor de uma grandeza física, independentemente da realização de uma medida, teríamos uma teoria física real. No entanto, em um sistema quântico tipo o experimento mental proposto em 1935 por Albert Einstein, Boris Podolsky e Natan Rosen (EPR), o critério de realidade física e de localidade, o qual estabelece que não é possível existir ações a distância ocorrendo instantaneamente ou com velocidade superior à da luz, ficaram comprometidos [13].

Veremos, agora, como o argumento EPR questionou a completude da mecânica quântica em relação a como Niels Bohr, um dos pais fundadores da interpretação usual da MQ, interpretou a função de onda $\psi$. Segundo ele, essa função possuía toda a informação necessária para descrever um sistema quântico. O próprio título do artigo, publicado na Physical Review, ilustra o que estava em jogo: Can Quantum-Mechanical Description of Physical Reality Be Considered Complete? Esse questionamento da completude da mecânica quântica culminou em uma das maiores controvérsias científicas do século XX [13].

A proposta do EPR vai de encontro a um dos princípios básicos da MQ usual: o princípio da incerteza, o qual apresenta que, na mecânica quântica, só existe a possibilidade de verificação simultânea e precisa das pro- 
priedades de um sistema, se os operadores associados a grandeza medida comutam entre si. A ideia apresentada por EPR consistia em considerar um sistema quântico com duas partículas correlacionadas em que a medição da propriedade de uma implicaria na obtenção da medida da segunda, mesmo que esta estivesse afastada espacialmente da primeira, e ainda que os operadores associados a grandeza medida não comutem entre si.

No argumento EPR, uma fonte emitia pares de partículas correlacionadas que se moviam em direções opostas. A medição da propriedade de uma partícula implicaria na obtenção da medida da segunda, mesmo estando espacialmente afastada da primeira. Utilizaremos aqui, para facilitar a compreensão do experimento de pensamento EPR, a versão discutida por Bohm a partir da ideia de spin - uma quantidade física intrínseca à cada partícula, da mesma forma que massa e carga, que pode ainda assumir valores numéricos [14]. Contudo, é importante destacar que EPR utilizaram originalmente um sistema de duas partículas, cujas propriedades utilizadas como observáveis foram posição e momento [13]. Ao realizar a medida do spin em um determinado eixo $(n)$ em uma das partículas, é possível obtermos o resultado de spin para cima $(+n)$ ou spin para baixo $(-n)$. De acordo com a interpretação da teoria quântica usual, a obtenção do resultado, através da medição, de spin para cima $(+n)$ em uma das partículas (A) colapsa o sistema quântico para um estado em que nos permite concluir que o spin da outra partícula (B) seja para baixo $(-n)$. Caso o spin obtido na partícula A seja para baixo $(-n)$, devemos atribuir o spin para cima $(+n)$ para a partícula $\mathrm{B}$, o qual não foi medido. Em outras palavras, o resultado do spin para a partícula B depende da medida efetuada na partícula A, havendo, assim, alguma correlação entre as medidas. Essa influência mútua resulta em um comportamento "não local" uma vez que, ainda que as partículas estejam espacialmente afastadas, a atuação de uma medida sobre a outra é instantânea [14].

Essas medições também podem ser realizadas ao longo do eixo $m$. No entanto, o spin do eixo $n$ e do eixo $m$ são observáveis chamadas de incompatíveis já que não é possível obter um valor definido para ambos os eixos. Se a medida for realizada em $n$, será impossível predizer com certeza a medida em $m$, até que esta seja realizada. A decisão, portanto, de escolher entre um eixo ou outro possui implicação na realidade física da observável no local da outra partícula. Ao se escolher o eixo $n$, a realidade da observável no eixo $m$ fica comprometida instantaneamente. Assim, o critério de realidade física e fenômenos com comportamentos não locais são incompatíveis.

Diante disso, temos duas hipóteses: ou as quantidades físicas associadas a dois operadores que não comutam entre si não apresentam realidade física; ou a mecânica quântica, através da função de onda, não fornece uma descrição completa da realidade. Esse comportamento, a princípio, era estranho - o que levou alguns físicos a questionarem a completude da mecânica quântica e, até mesmo, se era uma teoria correta [15].

Em outras palavras, o argumento EPR questionou a ideia do antirrealismo ao enfatizar que um sistema físico real existindo independente das condições de observação não é possível, levando, assim, a perda da noção de realidade física. Apenas seria possível a existência a partir da observação. O restabelecimento de uma teoria completa viria, por exemplo, a partir da restauração do determinismo através de variáveis adicionais [12]. Nesse sentido, a estrutura do argumento EPR admitiu o formalismo da MQ para a situação de duas partículas correlacionadas em seu caráter não local, bem como a hipótese de localidade associada à concepção de realidade de uma teoria física. O argumento EPR concluiu que a mecânica quântica não satisfaz a condição de completeza. Para que o formalismo da MQ seja considerado completo, seria necessário o abandono da condição de localidade ou da ideia de que a MQ seja completa [16].

Neste ponto, o questionamento referente à teoria quântica se faz evidente se considerarmos o critério da completude apresentado no artigo EPR, no qual uma teoria física seria completa somente se "todo elemento da realidade física tem uma contrapartida na teoria física"[13]. Pessoa Jr. apresenta o argumento EPR e as suas diversas interpretações considerando, ou não, a localidade e a completude de uma teoria. A interpretação ondulatória, por exemplo, é capaz de abandonar a condição de localidade e permanecer coerente, considerando, portanto, que a mecânica quântica é uma teoria completa [16].

Argumentos a respeito de fenômenos não locais são, de fato, um divisor de águas na compreensão dos fundamentos da MQ. O argumento EPR abordou uma experiência de pensamento que ainda não havia sido verificada nos laboratórios. Foi, nesse contexto, que surgiu em 1957 o trabalho de Bohm e Aharonov [7], o qual utilizou o experimento WS de 1950 como uma prova para o EPR, e que estava de acordo com a MQ.

\section{O experimento WS}

No ano de 1949, Wu e Shaknov concluíram o artigo que foi publicado no ano de 1950 e ficou conhecido como o experimento WS. O artigo começou tratando a proposta de 1946 do fisco teórico John Archibald Wheeler (1946) ${ }^{1}$ de realizar um experimento para verificar a predição da teoria de pares de Paul Dirac (1930) ${ }^{2}$ em que dois quantas (fótons) emitidos a partir da aniquilação do par elétronpósitron seriam polarizados em ângulos retos [17]. Esta era uma previsão da eletrodinâmica quântica e o objetivo de Wu era fazer sua verificação [18]. Os fótons eram obtidos após a colisão de um elétron e de um pósitron que se aniquilam, produzindo, deste modo, fótons de radiação gama e preservando as leis de conservação da

1 J.A. Wheeler, Annals of the New York Academy of Sciences 48: 219-238 (1946).

2 P.A.M Dirac, Camb. Phil. Soc. 26: 361-375 (1930). 
carga, do momento linear e angular. Para colidir o elétron com o pósitron, Wu utilizou um acelerador de partículas - o cíclotron - da Universidade de Columbia.

No artigo publicado na Physical Review, Wu iniciou discutindo os resultados experimentais obtidos anteriormente por Ernst Bleuler e H. L. Bradt em $1948^{3}$ na Universidade de Indiana nos EUA. Eles utilizaram contadores Geiger-Muller (GM) e obtiveram uma taxa de assimetria que estava dentro dos limites colocados pela teoria quântica. Porém, os experimentos possuíam uma margem de erro relativamente grande e isso comprometeria uma análise mais refinada entre teoria e experimento [17]. Apesar de possuir compatibilidade com a MQ, a taxa de incerteza de seus resultados era tão grande que os tornava inconclusivos [19].

Também citou o experimento de R. C. Hanna (1948) realizado no laboratório Cavendish da Universidade de Cambridge na Inglaterra, e ainda que utilizando contadores mais eficientes, resultados obtidos eram diferentes do esperado. Gouesbet (2013) apontou os resultados encontrados por Hanna (1948) como incompatíveis com a MQ. Enquanto o resultado dos experimentos de Hanna estava com uma taxa de aproximadamente de $10 \%$ a $28 \%$ abaixo do resultado previsto teoricamente, os resultados de Bleuler e Bradt apresentava um valor de aproximadamente $12 \%$ acima do previsto teoricamente [20]. Por conta das incoerências nos trabalhos anteriores, Wu justificou a necessidade de realização de outro experimento, desta vez, utilizando detectores mais eficientes e condições mais favoráveis [17].

O aparato WS consistia em um bloco de chumbo em que dutos colimavam a radiação emitida. No centro, a fonte de radiação emitia pares de fótons, e eram detectados nas extremidades por fotomultiplicadores (RCA 5819) que convertiam a detecção dos fótons em pulsos de corrente elétrica. WS utilizaram cristais de antraceno associados aos fotomultiplicadores por serem mais eficientes, o que possibilitou valores mais favoráveis na detecção da radiação. A Figura 1 apresenta o esquema do aparato experimental publicado no artigo de 1950.

O experimento WS consistia em obter medidas da taxa de coincidência de espalhamento de dois fótons de aniquilação. Enquanto um detector era mantido fixo, outro detector foi posicionado em ângulos de $0^{\circ}, 90^{\circ}, 180^{\circ} \mathrm{e}$ $270^{\circ}$ em relação ao primeiro. Wu e Shaknov observaram uma taxa de coincidência de detecção de fótons da ordem de quatro por minuto para ângulos perpendiculares [17]. A razão entre o número de detecção obtida nos detectores, dispostos perpendicularmente, fornecia a taxa de coincidência. Para essa disposição, a taxa de coincidência obtida no experimento foi de $2.04+/-0.08$ (em que 0.08 é o erro médio associado à medida), o que parecia bastante satisfatório já que a previsão teórica era de um valor máximo de 2,85. O tempo de duração para a realização do experimento foi de 30 horas contínuas,

\footnotetext{
3 E. Bleuler, H. L. Bradt, Phys. Rev. 73, 1398 (1948).

4 R.C Hanna, Nature 162, 332 (1948).
}

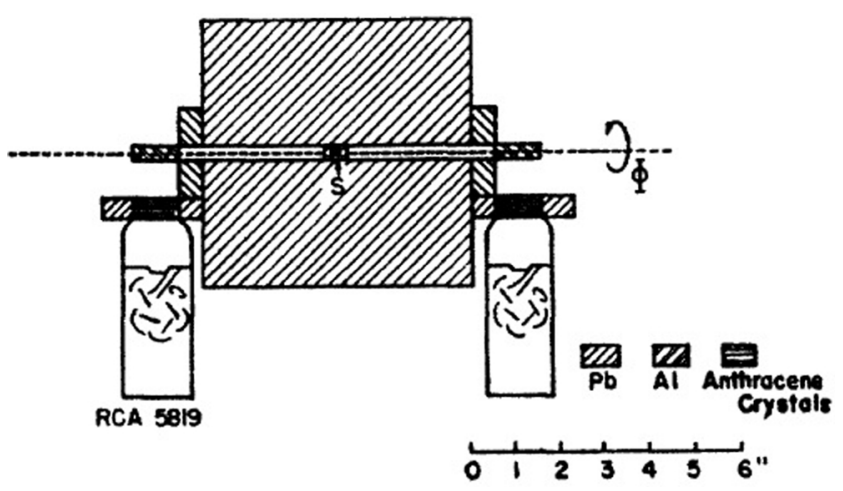

Figura 1: Aparato do experimento WS. Fonte: Wu e Shaknov (1950, p. 1).

o qual foi realizado no Laboratório Pupin de Física da Universidade de Columbia em New York.

É importante destacar que, quando chegou em 1944 à Universidade de Columbia, ainda vinculada ao projeto Manhattan, $\mathrm{Wu}$ trabalhou no aprimoramento do contador de partículas radioativas - o contador GM, e desenvolveu um contador de cintilação com eficiência cerca de dez vezes maior do que o GM. O aprimoramento e a utilização de um contador de cintilação mais eficiente, no experimento WS, foi imprescindível para possibilitar a obtenção de resultados que corroboravam a teoria de pares de Dirac, diminuindo, assim, significativamente o valor associado à medida para a margem de erro 0,08 [17]. Os dados obtidos no experimento corroboraram a hipótese de que dois fótons de radiação emitidos a partir da aniquilação elétron pósitron seriam polarizados em ângulos retos, garantido, então, alguma relação entre eles.

O experimento de $\mathrm{Wu}$ e Shaknov, apresentado em um artigo de uma única página, foi extremamente eficaz a ponto de não mais precisar de discussões futuras a respeito da previsão da eletrodinâmica quântica que ela havia se proposto a verificar [18].

\section{A relevância do experimento WS para o trabalho de Bohm}

Em detrimento da interpretação de Copenhague, na década de 50, surgiu uma proposta de reinterpretação da mecânica quântica a partir dos trabalhos do físico teórico norte americano David Bohm [1,21]. A sua ideia era estabelecer uma teoria determinística para a MQ, análoga à da Mecânica Clássica. Na teoria quântica, associada à interpretação de Copenhague, o princípio da incerteza apresenta a impossibilidade de determinar simultaneamente duas propriedades de um sistema quântico, como momento e posição, impedindo, assim, a sua classificação enquanto uma teoria determinística. No entanto, ao apresentar uma interpretação alternativa à interpretação de Copenhague, Bohm queria assegurar que a teoria quântica pudesse ser determinística [1] p. 21. Segundo 
ele, esta interpretação "fornece uma estrutura conceitual mais ampla do que a interpretação usual, porque possibilita uma descrição precisa e contínua de todos os processos, mesmo no nível atômico" [1] p. 21.

Um grande feito de Bohm foi apresentar em 1952 uma interpretação causal, alternativa à interpretação de Copenhague, a partir de variáveis adicionais. O seu argumento possuía implicações filosóficas que criticavam a interpretação usual da MQ, acusando-as de negligenciar a possível existência de propriedades que, até o momento, não eram possíveis de serem observadas. A sua crítica era em torno do caráter positivista e empirista da MQ usual. Em contrapartida, Bohm defendia o realismo descartado pela complementaridade. A sua proposta teórica foi capaz de levar às mesmas previsões da MQ usual, inserindo variáveis no sistema quântico e nos aparelhos de medida, sendo, portanto, uma interpretação alternativa à ideia de complementaridade $[1,6]$.

Bohm, então, apresentou um modelo físico capaz de reproduzir os mesmos resultados da teoria quântica usual. No entanto, o seu quadro conceitual reivindicava parâmetros adicionais para sustentar o determinismo do seu modelo [21]. Diferentemente da interpretação probabilística, vinculada ao princípio da incerteza de Heisenberg, a qual não permite uma medição simultânea de variáveis como momento e posição, no modelo físico de Bohm, é possível obter informação de tais variáveis de uma maneira bem definida e existindo simultaneamente. Contudo, isso aparece apenas na forma do que ele chamou de potencial quântico - um terceiro termo que aparece na equação da energia mecânica total do sistema juntamente com a energia cinética e potencial. Enquanto que, na interpretação usual, a função de onda $\psi$ define apenas a densidade de probabilidade, na interpretação de Bohm, a função $\psi$ também influencia a dinâmica da partícula através do potencial quântico [22]. Portanto, exige uma interpretação diferente daquela utilizada pela MQ usual.

A reação negativa dos físicos à proposta de Bohm apareceu, ainda em seu rascunho, por físicos como Wolfgang Ernst Pauli [1] p-32 e, após a publicação, com fortes críticas dos físicos Bohr e Léon Rosenfeld que eram defensores da mecânica quântica ortodoxa. No entanto, o apoio à sua proposta também teve nomes como Louis De Broglie e Jean-Pierre Vigier, além do físico argentino Mario Bunge e do brasileiro Jayme Tiomno [1]. A interpretação da mecânica quântica de Bohm foi importante por suscitar debates em torno da interpretação da teoria quântica, o que durou por muito tempo.

Em meados da década de 30, a maioria dos físicos não considerava que as questões associadas a fundamentos da MQ mereciam muita atenção, ou que o que realmente precisava ser discutido em termos de fundamentos já havia sido feito por Bohr e outros físicos [1]. O historiador Helge Kragh [4] argumenta que a grande maioria dos físicos parecia desinteressada em resolver problemas filosóficos, e que esse tipo de trabalho não tinha relevância para as pesquisas que eles estavam realizando. Foi apenas na década de 60 que o debate em torno do EPR se tornou mais conhecido. O artigo EPR está entre os dez mais relevantes trabalhos já publicados pela Physical Review, sendo considerado um argumento central para os debates que se desenvolveram, e que ainda permanecem, a respeito da teoria quântica [23].

Na tentativa de sustentar empiricamente os seus argumentos, Bohm encontrou no experimento de WS uma prova experimental que colocava em prática as ideias contidas no EPR, as quais surgiram inicialmente como uma experiência de pensamento. Como vimos anteriormente, Bohm havia em 1951 apresentado uma versão mais inteligível para o EPR, utilizando uma única quantidade física, que se apresenta quando a partícula é submetida a um campo magnético, o spin [23]. Utilizou, então, essa única variável associada a cada uma das partículas, as quais estariam separadas uma da outra, de maneira que a medida do spin de uma partícula pudesse ser verificada sem interferir na outra partícula. Após a versão de Bohm referente ao EPR, alguns autores acrescentam ao acrônimo EPR a letra B para se referir à versão bohmiana, a qual possibilitaria mais facilmente a realização de um experimento em laboratório, passando, assim, a ser conhecida como EPRB [15].

Com base nos dados cientométricos, nenhum dos onze artigos que citaram o experimento de Wu de 1950 até o ano de 1957, havia se preocupado em discutir o EPR ou a questão das variáveis ocultas. Depois de sete anos, o experimento de $\mathrm{Wu}$ foi se apropriado pela área de fundamentos da teoria quântica. Bohm, juntamente com o seu assistente de pesquisa Yakir Aharanov, ambos na Universidade de Haifa, em Israel, discutiu a ideia de que os fótons do experimento WS estavam em um estado de entrelaçamento. Ou seja, WS tinham produzido em 1950 a primeira evidência de fótons entrelaçados a partir da aniquilação de pósitrons. No entanto, o impacto do resultado experimental WS nos fundamentos da MQ apenas foi percebido tardiamente em 1957 pelo trabalho de Bohm e Aharonov [15,18].

Para além da questão de fótons entrelaçados, Bohm e Aharonov [7] apresentaram ainda o experimento de $\mathrm{Wu}$ como a primeira prova experimental para verificar a experiência de pensamento do EPR proposto há duas décadas. Em suas palavras,

[...] é demonstrado que já existe uma experiência cujo significado em relação a este problema ainda não foi explicitamente publicado, mas que é capaz de provar que essa resolução sugerida do paradoxo (bem como uma classe muito ampla de tais resoluções) não é sustentável. Assim, esta experiência pode ser considerada como a primeira prova empírica clara de que os aspectos da teoria quântica discutidos por Einstein, Rosen e Podolsky representam propriedades reais da matéria. [7], p. 1 - tradução nossa). 
Nesse trabalho, Bohm apresentou os dados obtidos por Wu e Shaknov em uma tabela, explicando o resultado experimental e associando-os a correlações à distância, como sugerido pelo argumento EPR. A sua interpretação do experimento WS, relacionando-o ao paradoxo EPR, repercutiu na comunidade científica e acabou impulsionando um debate em torno da real possibilidade de verificação do EPR a partir do experimento WS. Três anos mais tarde, em 1960, Asher Peres e P. Singer, do Instituto de Tecnologia em Israel, publicaram um artigo, intitulado On possible experimental tests for the paradox of Einstein, Podolsky and Rosen, no qual apresentavam que o argumento de Bohm em relação ao experimento de WS não era válido e propuseram outros experimentos:

\begin{abstract}
Há algum tempo, Bohm e Aharonov alegaram que a experiência de $\mathrm{Wu}$ e Shaknov (na correlação de polarizações de fótons de aniquilação) pode ser considerada como uma prova empírica contra a hipótese de Furry. No entanto, uma análise cuidadosa das propriedades dos fótons mostra que eles não são adequados para formular o paradoxo de Einstein, Podolsky e Rosen, de modo que o argumento de Bohm e Aharonov contra a hipótese de Furry não é válido. Alguns outros testes experimentais possíveis são propostos. [24] p. 1 - tradução nossa).
\end{abstract}

Sobre as críticas de Peres e Singer, Bohm afirmou que "são mostradas como errôneas baseadas em uma interpretação incorreta da polarização da radiação eletromagnética no domínio quântico [25] p. 1 - tradução nossa). Na visão de Bohm, o experimento WS mostrava um caminho para novas possibilidades de experimentação que conduziriam os físicos experimentais a encontrarem respostas para questões da teoria quântica, a exemplo da não localidade e da teoria das varáveis ocultas locais. O paradoxo EPR foi revisitado novamente com o trabalho teórico de John S. Bell (1964) $)^{5}$ que indicava a possibilidade de verificação da existência, ou não, de variáveis escondidas que supostamente completariam a MQ. Os experimentos que testaram as desigualdades de Bell foram realizados posteriormente por nomes como John Clauser, Abner Shimony, Michael Horne, Alain Aspect, Stuart Jay Freedman, entre outros.

Apesar do experimento WS não servir enquanto uma prova experimental para a verificação das variáveis ocultas, ele contribuiu significativamente enquanto precursor dos experimentos envolvendo o fenômeno do entrelaçamento, servindo de ponto de partida para os físicos experimentais já que o experimento apresentava a questão da não localidade e corroborava a mecânica quântica. A comprovação mais conclusiva do fenômeno do entrelaçamento quântico veio com os trabalhos de John Clauser

5 Bell, J.S. On the Einstein-Podolsky-Rosen paradox. In: Physics, 1: 19-200, 1964. e Freedman em 1972 [15]. A contribuição de tais experimentos e outros levaram a avanços que resultaram na segunda revolução quântica.

\section{A segunda revolução quântica}

O fenômeno do entrelaçamento quântico se mostra tão relevante para a ciência que o físico francês Alain Aspect, premiado por seus trabalhos experimentais associados ao EPR e o teorema de Bell, cunhou o termo segunda revolução quântica por considerar a década de 60 como um momento de mudança na física e de grande avanço tecnológico [1]. A habilidade experimental dos físicos manipularem individualmente sistemas quânticos, tal como elétrons e fótons, também faz parte desta revolução na física. Entretanto, concordamos com Freire [1] ao discutir as origens desta segunda revolução a partir da década de 50 quando interpretações alternativas à de Copenhagen ganharam notoriedade, principalmente a partir do trabalho de físicos considerados como dissidentes. E destacamos, ainda, a contribuição do que ficou conhecido como a primeira geração de experimentos que tratavam do fenômeno.

De maneira semelhante à primeira revolução quântica, a qual se refere ao próprio advento e desdobramentos da MQ em meados da década de 20 , a segunda revolução quântica também teve seu desenvolvimento de maneira gradual. A própria cultura material, associada às técnicas e à instrumentação disponível à época para a obtenção de resultados significativos, teve seu avanço gradualmente. Se pensarmos na segunda revolução quântica com base nos experimentos, tendo o fenômeno do entrelaçamento enquanto um dos principais elementos a ser desenvolvido, é perceptível a fragmentação desta teoria em algumas gerações de experimentos. O grande motivador a apresentar os experimentos que contribuíram para a segunda revolução quântica divididos em gerações é a forma com que os pares de fótons emaranhados são produzidos, além das técnicas de detecção e do aparato experimental que se desenvolveu ao longo dos anos, possibilitando resultados cada vez mais conclusivos.

A primeira geração dos experimentos apresenta os primeiros resultados no final da década de 40, com os trabalhos de Bleuler (1948) Hanna (1948) e Vlasov (1949), avançando até a década de $50 \mathrm{com}$ os trabalhos de WS (1950), Hereford (1951) e Bertolini (1955). Para Gouesbet, essa geração é considerada a geração zero dos experimentos que chegaram ao fenômeno do entrelaçamento a partir da radiação de aniquilação e serviriam de base para experimentos futuros. Eram experimentos que corroboraram a MQ. No entanto, a corroboração de uma teoria não é necessariamente capaz de validá-la [17]. A segunda geração obteve o par de fótons emaranhados a partir de laser sintonizável que excitavam as amostras atômicas, tendo Aspect e E. Fry como protagonistas [26]. Essa, em detrimento à primeira, se volta às questões da MQ envolvendo o fenômeno do entrelaçamento relacio- 
nado ao EPRB, e à verificação experimental da Teoria das Variáveis Ocultas Locais (TVOL). A terceira geração, já identificada por experimentos voltados para testar as desigualdades de Bell, a partir de 1988, produzia fótons emaranhados a partir da interação de fótons emitidos por laser interagindo com cristais não lineares [26].

$\mathrm{Na}$ década de 60, uma família de resultados teóricos que mostravam a impossibilidade de interpretação da MQ a partir de uma concepção realista local foi apresentada e ficou conhecida como Teorema de Bell. O nome está associado ao físico teórico irlandês John Stewart Bell que propôs esses resultados no trabalho de 1964 e que foi capaz de transformar os estudos referentes à teoria quântica [27]. O trabalho de Bell consistia em apresentar que a MQ não poderia ser interpretada a partir das TVOL, pois esta configuração violaria as desigualdades que foram apresentadas em seu trabalho teórico. Em outras palavras, se a MQ fosse correta, partículas entrelaçadas deveriam satisfazer ao teorema de Bell [19]. Os primeiros testes experimentais que buscaram testar as desigualdades de Bell apresentaram resultados mais conclusivos a respeito da necessidade ou não de utilização da TVOL que completariam a MQ, conforme apresentado no EPR. Os testes experimentais foram realizados inicialmente por Clauser et al. (1969) e Horne (1974) $)^{7}$, Aspect (1983) ${ }^{8}$ e Mermin (1986) ${ }^{9}[27]$.

O teorema de Bell se apresenta enquanto um marco para teoria quântica, inclusive desempenhou um papel indispensável na segunda revolução quântica. O teorema de Bell e os experimentos que a partir dele se desenvolveram possibilitaram um novo campo de estudo da física que começava a se consolidar: a informação quântica. Whitaker [18], ao discutir os primeiros responsáveis pelos experimentos que levaram Bell a formular a sua teoria quântica, destacou o experimento de Wu e Shaknov como o primeiro a produzir feixes de fótons entrelaçados.

Whitaker indicou o experimento de Wu localizado na primeira geração de experimentos que conduziu ao entrelaçamento [18], e apresentou como as ideias teóricas de Bohm influenciaram o teorema de Bell. Se estabelecermos um análogo em termos do desenvolvimento do aparato experimental utilizado para verificar o teorema de Bell, é percebida a contribuição do trabalho de Wu nos experimentos de Clauser e Shimony, os quais chegaram a indicar o experimento WS enquanto a primeira referência para se debruçar sobre os experimentos associados ao teorema de Bell.

\footnotetext{
${ }^{6}$ Clauser, J.F., Horne, M.A., Shimony, A., and Holt, R.A. Proposed experiment to test local hidden-variable theories. Physical Review Letters, 23: 880-884, 1969.

7 Clauser, J.F. and Horne, M.A. Experimental consequences of objective local theories. In: Physical Review D, 10: 526-535, 1974. ${ }^{8}$ Aspect, A.Trois tests expérimentaux des inégalités de Bell par mesure de corrélation de polarization de photons, Orsay: Thèsed'Etat, 1983.

9 Mermin, N.D. Generalizations of Bell's Theorem to higher spins and higher correlations. In: Fundamental Questions in Quantum Mechanics, L.M. Roth and A. Inomato (eds.), New York: Gordon and Breach, 7-20, 1986.
}

O físico norte americano John Clauser obteve o seu título de doutorado na Universidade de Columbia, onde $\mathrm{Wu}$ trabalhava enquanto pesquisadora e docente. Inicialmente começando a trabalhar de maneira isolada, diferente de Shimony que tinha a colaboração de Michael Horne e Richard Holt, Clauser buscou uma configuração experimental para testar a proposta teórica das desigualdades de Bell que revelariam, ou não, a existência de variáveis escondidas como sugerido no artigo EPR. No entanto, em 1964 Bell não deixou claro os detalhes técnicos de como realizar um experimento daquela natureza. Clauser então decidiu buscar experimentos que poderiam ter sido ignorados por Bell e que ajudariam a compreender a configuração experimental. O único trabalho que encontrou, que indicava um caminho, foi o experimento WS [17], embora não abordasse de maneira completa o problema da correlação.

Em Columbia, Clauser chegou a discutir com Wu o experimento WS. A sua grande dúvida era se Wu havia realizado as medidas de correlação entre os fótons para outros ângulos, além dos que foram apresentados no trabalho [15]. A consulta de Clauser a Wu foi importante, pois, caso ela tivesse realizado as medidas de polarização para ângulos intermediários, ele poderia ter os resultados experimentais que poderiam testar as desigualdades de Bell. Essas medidas, no entanto, não foram realizadas por $\mathrm{Wu}$, pois os fótons obtidos a partir da aniquilação pósitron-elétron possuíam alta energia, não sendo capaz de fornecer informações suficientes referentes à polarização entre pares.

Wu então indicou a Clauser o seu estudante de pósgraduação, Leon Kasday, que estava refazendo seus experimentos. Os resultados do experimento foram publicados em 1975 por Kasday com a colaboração de Wu e Ullman [28]. O experimento foi capaz de corroborar a mecânica quântica, contudo, as correlações não foram explicitamente medidas, havendo a necessidade de inferir pressupostos auxiliares, não necessariamente verificados, o que acabou por enfraquecer seus resultados. Mesmo assim, argumentam que, apesar de não produzir evidências capazes de testar as desigualdades de Bell, o experimento apresentou fortes evidências contra a Teoria das Variáveis Ocultas [29].

De maneira semelhante a Clauser, Horne, estudante de doutorado que trabalhava com Shimony, também revisitou os trabalhos de Wu. Até a chegada de Horne, Shimony havia avançado muito pouco nos estudos referentes ao teorema de Bell. Tanto Shimony quanto Horne consideravam a teoria quântica e as sugestões de testes experimentais como contribuições das mais importantes para física. Através da interação com Shimony, Horne conheceu dois artigos de Bell. Ele ficou tão entusiasmado que Simony o convidou para realizar experimentos do tipo EPRB, começando, assim, uma parceria no estudo dos fundamentos da MQ $[18,1]$

Uma das primeiras tarefas de Horne foi buscar experimentos da primeira-geração para verificar se tais experi- 
mentos mantiveram a posição dos detectores inalterada. Outra busca foi pelos experimentos da segunda geração que deveriam fixar a posição dos detectores enquanto as partículas entrelaçadas estivessem em voo. Horn revelou que "a primeira coisa que fiz depois de receber a missão de Abner [Shimony] foi olhar os resultados de Wu e Shaknov" [15] p.159-160. Mais uma vez, o trabalho de Wu se mostrou relevante para a área de fundamentos. Porém, Horne também percebeu que com o experimento WS era difícil verificar a polarização dos fótons por conta da sua alta energia. Para ele, realizar os experimentos na faixa da luz visível traria resultados mais satisfatórios.

Para aumentar a colaboração em torno das investigações referentes à teoria quântica, Shimony convidou Clauser a trabalhar com ele e o seu grupo de pesquisa. O fruto dessa colaboração foi um artigo publicado por Clauser, Horne, Simony, e Holt, no qual propuseram um experimento para testar a consistência entre a MQ e a TVOL, o qual ficou conhecido pelo acrônimo $\mathrm{CHSH}^{10}$ $\mathrm{O}$ artigo adequava o teorema de Bell a um experimento passivo de ser realizado [1]. Os primeiros físicos a apresentar resultados experimentais bem-sucedidos referente ao teorema de Bell foram o próprio Clauser e Stuart Jay Freedman em 1972, oito anos depois da proposta teórica $[26]$.

Mais recentemente, o experimento WS de 1950 foi reconhecido como o precursor do entrelaçamento quântico. Em 2012, o físico chileno Francisco Javier Duarte, premiado em 1995 por suas contribuições à engenharia ótica pelo Paul F. Forman Engineering Excellence Award e laureado em 2016 com a medalha David Richardson da Optical Society of America, publicou o artigo The origin of quantum entanglement experiments based on polarization measurements [20] no qual discutiu os artigos que surgiram no final da década de 40 que estavam associados ao fenômeno de entrelaçamento quântico, mas que ainda são frequentemente ignorados ou não referenciados, a exemplo do experimento de Wu e Shaknov.

Duarte também apresentou um panorama dos trabalhos mais citados, começando pelo renomado artigo de 1935 [13], passando pelo trabalho de Bohm e Aharonov em 1957, e finalmente chegando ao trabalho de Bell em 1964. Duarte [20] apontou como origem dos trabalhos teóricos sobre entrelaçamento quântico o artigo Polyeletron $\$$ publicado por John Archibald Wheeler, no qual faz referência a teoria de pares proposta em 1930 por Paul Dirac. A partir desse trabalho, Wheeler apresentou a essência do entrelaçamento quântico. Em 1949, Ward apontou uma negligência associada a uma derivação que apresentava valores discrepantes. O mesmo erro foi percebido, independentemente de Ward, por Simon Pasternack e John Hornbostel [20]. Naquele momento, o

10 CLAUSER, John F. et al. Proposed Experiment to Test Local Hidden Variable Theories. In: Physical Review Letters, v. 24, n. 10, p. 549,1970 .

${ }^{11}$ WHEELER, John Archibald. Polyelectrons. Annals of the New York Academy of Sciences, v. 48, n. 3, p. 219-238, 1946. esquema teórico estava apresentado. Entretanto, a pendência era realizar os experimentos. Em 1948, os físicos R. C Hanna e Ernst Bleuler, juntamente com H. L. Bradasse, debruçaram-se sobre a teoria e realizaram os experimentos. $\mathrm{O}$ detalhe é que os experimentos apresentaram valores afastados do que estava previsto teoricamente. A comprovação experimental apenas viria em 1950 com o trabalho WS [20].

\section{Considerações finais}

A presença das mulheres na ciência é historicamente minoritária em relação aos homens. Ademais, suas contribuições são frequentemente ofuscadas e/ou negligenciadas, quando não inteiramente apropriadas por personagens masculinos. Compreender os motivos pelos quais as mulheres não são percebidas na ciência é ainda o foco de investigação de diversas pesquisadoras. Apresentar uma história da ciência envolvendo a participação de mulheres é uma maneira de dar visibilidade à contribuição de mulheres para a ciência no sentido de garantir mais argumentos voltados para discussões de gênero. Ao observarmos livros que tratam da história da MQ, a exemplo dos livros de Helge Kragh [4]; Max Jammer [5] e Olival Freire [1], não percebemos a preocupação dos autores em apresentar o experimento de Wu enquanto relevante para a história da MQ. E, quando o fazem, limitam-se apenas a citar o experimento, sem considerar a sua repercussão.

Conforme discutido, o primeiro experimento associado ao fenômeno do entrelaçamento quântico foi desenvolvido por uma mulher dentro de uma área de pesquisa majoritariamente masculina. O Experimento WS foi ainda, segundo a argumentação de Bohm, uma prova para o EPR. Bohm pôde, assim, contribuir para o desenvolvimento do conhecido Teorema de Bell que propunha testes experimentais para verificar a validade da MQ. Como destacamos, os primeiros testes experimentais tinham como referência o trabalho de $\mathrm{Wu}$ de 1950 . Foi, portanto, nesse contexto que o fenômeno do entrelaçamento quântico ganhou notoriedade servindo como principal elemento para o desenvolvimento de um novo campo de pesquisa, a informação quântica, derivado da segunda revolução quântica.

Observarmos $\mathrm{Wu}$ enquanto uma figura feminina na física com diversas contribuições, inclusive na mecânica quântica. É importante questionarmos ainda a predominância de mulheres atuando na física experimental em detrimento da física teórica, não no sentido de atribuir critérios de importância, mas de verificar até que ponto a comunidade científica reconhece e/ou aceita mulheres trabalhando em física teórica. A habilidade experimental de $\mathrm{Wu}$, vinculada à capacidade de verificar experimento com teoria, lhe garante uma posição relevante ao tratarmos da história da física e, consequentemente, da história da mecânica quântica.

Ainda assim, entretanto, destacamos a desvalorização do experimento de $\mathrm{Wu}$ por parte de historiadores da 
teoria quântica, cuja atenção é voltada para personagens tradicionais, e consequentemente, mais uma mulher e sua contribuição à física - foi deixada de lado pela historiografia tradicional. Para garantir à ciência um status mais justo e representativo, a história das mulheres precisa ser contada.

\section{Referências}

[1] O. Freire, The Quantum Dissidents: Rebuilding the Foundations of Quantum Mechanics 1950-1990 (Springer, Heidelberg, 2014).

[2] I. Silva, O. Freire Jr. and A.P.B. Da Silva, Revista Brasileira de Ensino de Física 33, 4601 (2011).

[3] M. Badino, Centaurus 58, 327 (2016).

[4] H. Kragh, Quantum generations: A history of physics in the twentieth century (Princeton University Press, New Jersey, 2002).

[5] M. Jammer, Philosophy of Quantum Mechanics: the interpretations of quantum mechanics in historical perspective (John Wiley and Sons, New York 1974).

[6] D. Bohm. Phys. Rev. 85, 2 (1952).

[7] D. Bohm and Y. Aharonov, Physical Review 108, 1070 (1957).

[8] R. Hammond, Chien-Shiung Wu: Pioneering Nuclear Physicist (Chelsea House Publishers, New York, 2009).

[9] T.C Chiang, Madame Chien-Shiung Wu: The First Lady of Physics Research (World Scientific, Singapura, 2014).

[10] J. Christian, in: Teoria quântica: estudos históricos e implicações culturais, editado por O. Freire, O. Pessoa and J. Bromberg (Eduepb, Campina Grande, 2011).

[11] N. Bohr, Nature 121, 580 (1928).

[12] M. Paty, in: Teoria quântica: estudos históricos e implicações culturais, editado por O. Freire Jr, O. Pessoa and J. Bromberg (Eduepb, Campina Grande, 2011).

[13] A. Einstein, B. Podolsky and N. Rosen, Physical Review 47, 777 (1935).

[14] D. Bohm, Quantum Theory (Prentice Hall, New York, 1951).

[15] A.D. Aczel, Entanglement: the greatest mystery in physics (Four Walls Eight Windows, New York, 2001).

[16] O. Pessoa Jr., Conceitos de Física Quântica (Livraria da Física, São Paulo, 2006) v. 2.

[17] C.S. Wu and I. Shaknov, Physical Review 77, 136 (1950).

[18] A. Whitaker, John Stewart Bell and Twentieth-Century Physics: Vision and Integrity (Oxford University Press, Oxford, 2016).

[19] G. Gouesbet, Hidden worlds in quantum physics (Courier Corporation, New York, 2013).

[20] F.J. Duarte, The European Physical Journal H 37, 311 (2012).

[21] O. Freire Jr, M. Paty and A.L.R. Barros, Estudos Avançados 8, 20 (1994).

[22] M.A.V. Macedo Jr, Tópicos atuais em física quântica: das ondas de matéria à realidade quântica. Dissertação de mestrado, Instituto Federal de Educação, Ciência e Tecnologia do Rio de Janeiro, Rio de Janeiro (2012).

[23] A. Fine, The Stanford Encyclopedia of Philosophy, disponível em: https://plato.stanford.edu/archives/ win2017/entries/qt-epr/, acessado em nov. 2017.

[24] A. Peres and P. Singer, Il Nuovo Cimento 15, 907 (1960).
[25] D. Bohm and Y. Aharonov, Il Nuovo Cimento 17, 964 (1960).

[26] W.F. Bispo, D.F.G. David and O. Freire, Revista Brasileira de Ensino de Física 35, 3603 (2013).

[27] A. Shimony, The Stanford Encyclopedia of Philosophy, disponível em: https://plato.stanford.edu/ archives/fall2017/entries/bell-theorem//acessado em nov. 2017.

[28] L.R Kasday, J.D Ullman and C.S Wu, Nuovo Cimento B 25, 633 (1975).

[29] F.J. Duarte, Quantum optics for engineers (CRC Press, New York, 2013). 